\title{
Automated system for ball launching, visual occlusion, and data acquisition in a ball-hitting task
}

\author{
KENNETH NEMIRE, MARK GOETTSCHE, and BRUCE BRIDGEMAN \\ University of California, Santa Cruz, California
}

\begin{abstract}
Investigations of the mechanisms underlying visual-motor coordination traditionally have been limited to static situations or one-dimensional motions. Technical advances are required to determine whether experimental results from these restricted behavior domains generalize to dynamic action in three dimensions, such as ball-hitting. In this paper, we describe electromechanical devices, under computer control, for projecting a table-tennis ball, selecting monocular or binocular visual input, and recording ball-hitting performance. The automated system allows freedom of movement for the batter, can be used in a moderately sized laboratory, and can be made from easily obtainable, inexpensive parts.
\end{abstract}

Our everyday activities include many examples of tasks requiring coordination of eye and hand, such as pointing at a distant point while giving directions and grasping a pen. Hitting or catching a baseball and executing a slam dunk are examples of visual-motor tasks from more athletic domains. Investigations of the mechanisms underlying skilled perceptual-motor coordination have been limited largely to static situations involving simple stimuli and simple responses and to tasks requiring one-dimensional motions (for review, see Schmidt, 1987). Appropriate technical advances are required to determine whether experimental results from these restricted behavior domains generalize to more complex action categories.

Some theoretical issues in understanding perceptualmotor behavior concern the characteristics of the afferent information capable of influencing motor behavior. Binocular and monocular contributions have been investigated (Bootsma \& van Wieringen, 1988; McLeod, McLaughlin, \& Nimmo-Smith, 1985), as have differences in central and peripheral retinal input (Davids, 1988; Paillard, 1980). Modifying the kind of information available during a perceptual-motor event can lead to a deeper understanding of the underlying mechanisms.

\footnotetext{
This work was completed while $K$. Nemire was satisfying the requirements for the doctoral degree in Experimental Psychology from the University of California, Santa Cruz. This research was supported by grants from the University of California, Santa Cruz to B. Bridgeman and to K. Nemire, and by a grant-in-aid of research from the Edmond and Marianne Blaauw Ophthalmology Fund of the National Academy of Sciences, through Sigma Xi, the Scientific Research Society to K. Nemire. We thank them for their assistance. The manuscript was completed while K. Nemire held a National Research Council/NASA-Ames Research Associateship. We also thank Michael Spivey for technical assistance and two anonymous reviewers for their helpful comments. Correspondence should be directed to Kenneth Nemire, NASA-Ames Research Center, Mail Stop 239-11, Moffett Field, CA 94035-1000.
}

Another issue concerns the extent to which sensory information can modify an ongoing rapid movement. Some evidence indicates that a minimal processing time is required for visual feedback to influence execution of movements (Carlton, 1981; Keele \& Posner, 1968; Zelaznik, Hawkins, \& Kisselburgh, 1983), while other results suggest that visual information is continuously available for control of rapid movements (Bootsma \& van Wieringen, 1990; Lee \& Young, 1984; Lee, Young, Reddish, Lough, \& Clayton, 1983). We can assess the functional relevance of various characteristics of the optic input to perceptualmotor coordination as well as the influence of the time of availability through analyses of relevant movement parameters, such as response and movement times, movement acceleration, and spatial location.

In this paper, we describe devices, made from easily obtainable parts, useful for projecting a table-tennis ball, selecting monocular or binocular visual input, and recording ball-hitting performance in the spatial and temporal domains. The instruments allow freedom of movement for the experimental participant, can be used in a moderately sized laboratory, and are relatively inexpensive to make. ${ }^{1}$ The equipment can be operated under computer control. We used a PDP/11-23 minicomputer with an RT11 operating system to automatically control ball firing, visual occluders, and paddle scanning required for data collection. The computer interface consisted of four analog input channels, three analog output channels, and four digital output channels. The computer program was written so that ball delivery could occur only when the paddle was in the ready position, and the state of each visual occluder (open or closed) could be changed prior to each trial.

The automated system described in this paper evolved from an investigation of visual and oculomotor contributions to perception and action in a ball-hitting task (Nemire, 1990). Because we were interested in subjects' ac- 
tions upon three-dimensional objects, we did not want to limit the experimental session to interaction with a CRT screen; the perceptual system, as indicated by a verbal judgment or a push of the button, may utilize different information sources than those used by the motor system in reaching or hitting tasks (Bridgeman, Kirch, \& Sperling, 1981; Bridgeman, Lewis, Heit, \& Nagle, 1979; Hansen \& Skavenski, 1985; Stark \& Bridgeman, 1983).

The subject's task in the experiment was to hit a tabletennis ball with the center of the paddle so that it hit a target directly in front of him. The system we describe consists of electromechanical devices that can be used separately or with other instruments, depending on the desired task. Where applicable, we describe other uses of the devices, as well as means of modifying them to accommodate other tasks.

\section{Ball Launcher}

Commercial devices that launch baseballs or tennis balls were not suitable for operation in a moderately sized laboratory. We opted to launch table-tennis balls. ${ }^{2}$

The ball-delivery device consisted of an automatic ball feeder and a ball launcher (Figure 1). The container held about 70 balls. A rotating arm at the bottom of the ballholding container forced the balls into a chute. A computercontrolled push solenoid (Electromechanisms, Inc., SP75$12 \mathrm{~V}$ ) ejected a ball from the chute onto the launching platform. Another computer-controlled push solenoid, provided with a $130-\mathrm{V}$ power source, launched the balls. Overdriving the solenoid did not burn it out since power was applied for only $20 \mathrm{msec}$ at a time, with pauses of at least $1 \mathrm{sec}$. The 20 -msec power pulse was $150 \mathrm{~V}$ peak to peak, supplying about $7 \mathrm{~A}$ of current. The power pulse was turned on when the power cycle was near $0 \mathrm{~V}$; an analog timing reference was used to monitor the power cycle.

The stock pushrod of the solenoid was replaced with a stainless steel rod, $3 \mathrm{~mm}$ in diameter and $34 \mathrm{~mm}$ in length, allowing greater launch accuracy and enhanced pushrod durability. During the power stroke, the pushrod struck the median plane of the ball $1 \mathrm{~cm}$ below the diameter after traveling $10 \mathrm{~mm}$ and continued another $14 \mathrm{~mm}$ after ball contact.

The launcher was positioned $105 \mathrm{~cm}$ above the floor at a vertical angle of $20^{\circ}$. Balls were delivered $474 \mathrm{~cm}$ to the actor's median plane at an approximate height of $105 \mathrm{~cm}$. About $95 \%$ of the balls were delivered to an area approximately described by a $25-\mathrm{cm}$-diameter circle. While the experimental design only required a firing frequency of six balls per minute, maximal firing frequency was 30 balls per minute. The solenoid delivered the balls so that mean velocity at the approximate plane of paddle interception was $7.1 \mathrm{~m} / \mathrm{sec}$.

With each ball launch, the plunger face sharply struck the solenoid casing. To prevent the subject from using the resultant auditory cue to time of ball onset, a second push solenoid (the false-firing solenoid, Figures 2 and 3 ) was randomly activated zero to nine times (the duty cy-

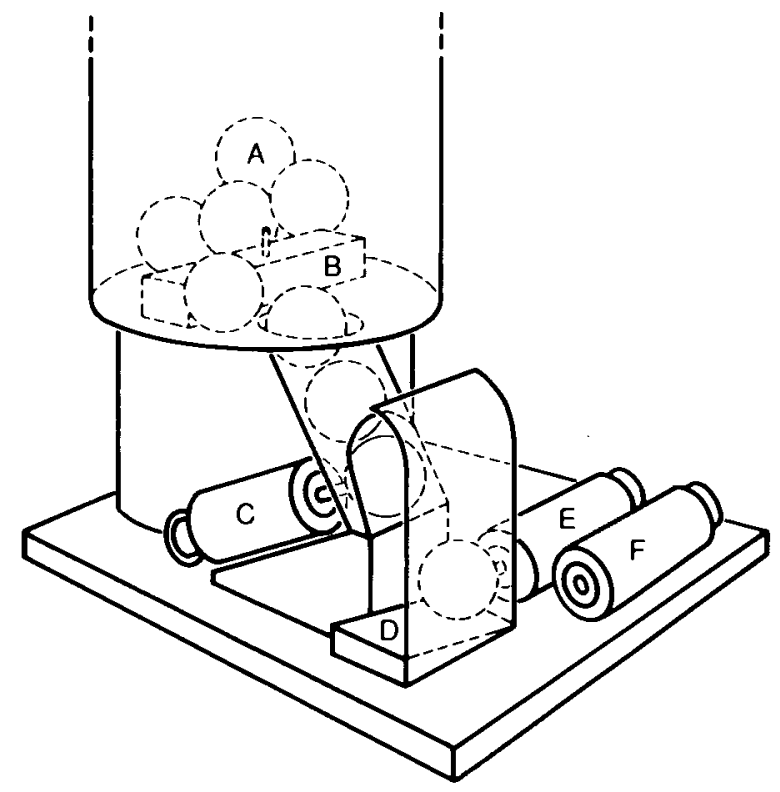

Figure 1. Perspective view of the apparatus used to project balls toward the experimental participant. (A) Balls in ball-holding container. (B) Rotating arm to move balls into chute. For optimal functioning, there must be enough space between the end of the rotating arm and the wall of the ball-holding container to allow easy passage of a ball. (C) Push solenoid (ball-feeder solenoid) to eject halls from chute onto launching surface (D). (E) Push solenoid to launch balls ( firing solenoid). (F) Push solenoid used to provide catch trials ( false-firing solenoid). Not depicted on the floor of the launching surface is the ball stopper, consisting of two small screws positioned one centimeter apart, to prevent the ball from rolling into the solenoid.

cle was 20 msec of "on" time and 1,000 msec of "off" time) prior to every ball launch. One second also elapsed between the last power stroke of the second solenoid and the power stroke of the ball-launching solenoid.

We present parameters of the ball-launching device used in our experiment. One may alter the capabilities of the system, such as firing rate and ball velocity, by using a different solenoid, input voltage, and/or duty cycle. Ball trajectory can be manipulated by changing the relationship of the ball and pushrod contact and by changing the angle of the ball-launching platform.

A block diagram of the ball-delivery subsystem is depicted in Figure 2. The schematic of the control circuitry for the two solenoids and the automatic ball feeder is shown in Figure 3.

\section{Paddle}

Hitting a ball to a target requires interception of the ball by the paddle at a precise moment in space and time. Spatial and temporal errors of interception can be recorded by noting the relative positioning of the ball and paddle contact from trial to trial. One method of collecting ballhitting responses has been to use trained observers or video equipment to collect the data and to infer type of batting error, such as undershooting or overshooting, from 


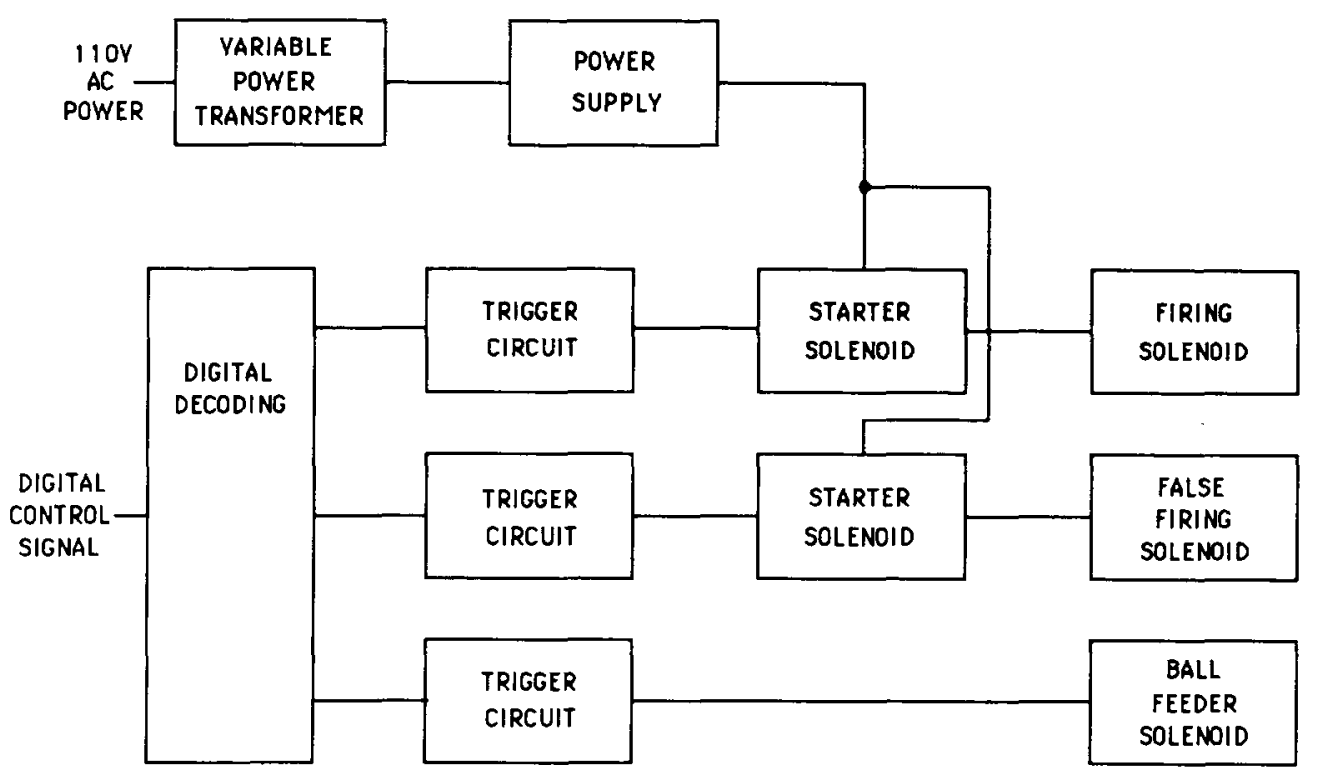

Figure 2. Block diagram of the circuit controlling the ball-delivery apparatus.

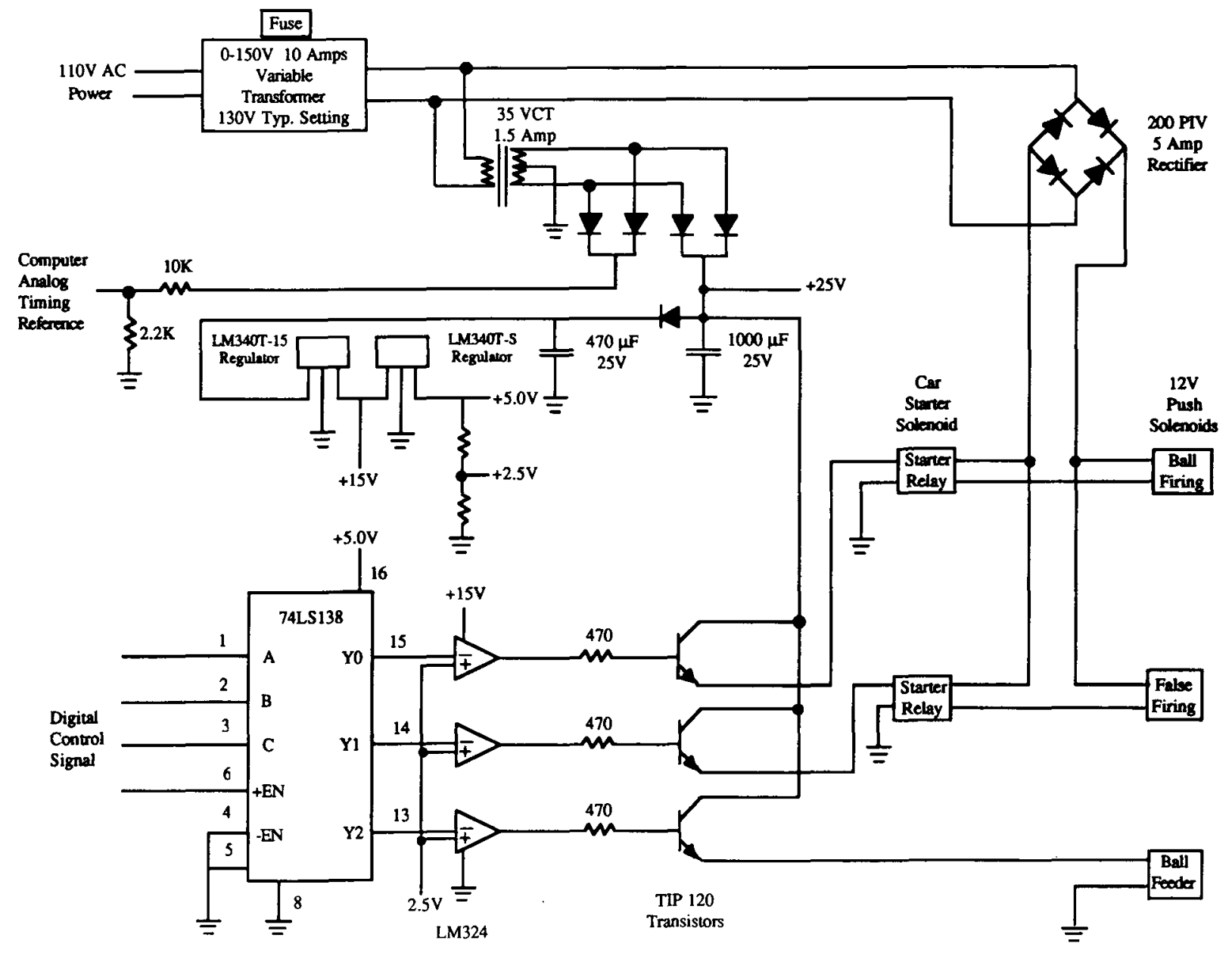

Figure 3. Schematic for the ball-delivery apparatus. 
the direction of the ball as it leaves the bat (DeLucia \& Cochran, 1985; McLeod et al., 1985; Shebilske, 1986; Smyth \& Marriott, 1982). We wanted, instead, to automatically record spatial and temporal interception errors as indicated by the location of the ball-paddle contact. We employed a paddle-mounted pendulum to record changes in response time and an infrared light grid on the paddle to record some spatial and temporal errors of the arm movement (Figure 4).

A pendulum was enclosed within the paddle such that when the paddle was held with the arm alongside the body and extended downward, the pendulum opened an infrared light switch (Figure 4). The ball was not launched until the pendulum was in the open-switch position. The switch closed when the subject began paddle movement towards the ball, indicating response latency.

The subjects initiated paddle movement from a constrained starting position; the computer program disallowed backswings. One could change pendulum parameters to fit other task requirements. The axis of the pendulum could be rotated to detect deviations from any other constrained starting positions. Also, to include preparatory and ballistic components of the swing, one or more accelerometers can be placed on the paddle to record acceleration of the paddle during the entire arm movement (Bootsma \& van Wieringen, 1990; Franks, Weicker, \& Robertson, 1985; Tyldesley \& Whiting, 1975).

An electronic circuit on the paddle described the spatial and temporal characteristics of the ball-paddle contact and sent the information to a digital computer. This information included the location and time of the ball-paddle contact position. Commercially available means of digitizing spatial location required either an electrical contact or radio, ultrasound, or pressure transduction. These interfaces were difficult to implement using table-tennis balls. We opted for an optic solution.

The paddle consisted of a sheet of $0.32-\mathrm{cm}$-thick plywood, $23 \times 31 \mathrm{~cm}$, with a handle. The effective paddle area for ball contact was $16.6 \times 20.3 \mathrm{~cm}$; the rest of the area housed the electronic circuit. The entire paddle weighed $0.79 \mathrm{~kg}$. The hitting surface and component housing could be constructed of lighter materials, if desired.

The location of ball-paddle contact was indicated by the interruption of a grid of infrared light beams delivered over the paddle surface. Eight infrared high-output LEDs were placed along one horizontal side of the paddle, and 10 LEDs were placed along one vertical side. Infrared sensor transistors were placed along the opposite sides to detect the infrared light beams. The LEDs were spaced $14 \mathrm{~mm}$ apart. The infrared beams were guided across the paddle through 5-mm apertures. The center of each aperture was placed $1.9 \mathrm{~cm}$ above the paddle surface. LED spacing was such that during contact, a table-tennis ball $(3.7 \mathrm{~cm}$ in diameter) always interrupted two beams in the two major axes of the paddle. Beam interruption was detected by sending the outputs of the infrared transistors to analog comparators, which in turn varied their digital output dependent upon beam interruption. "Low"
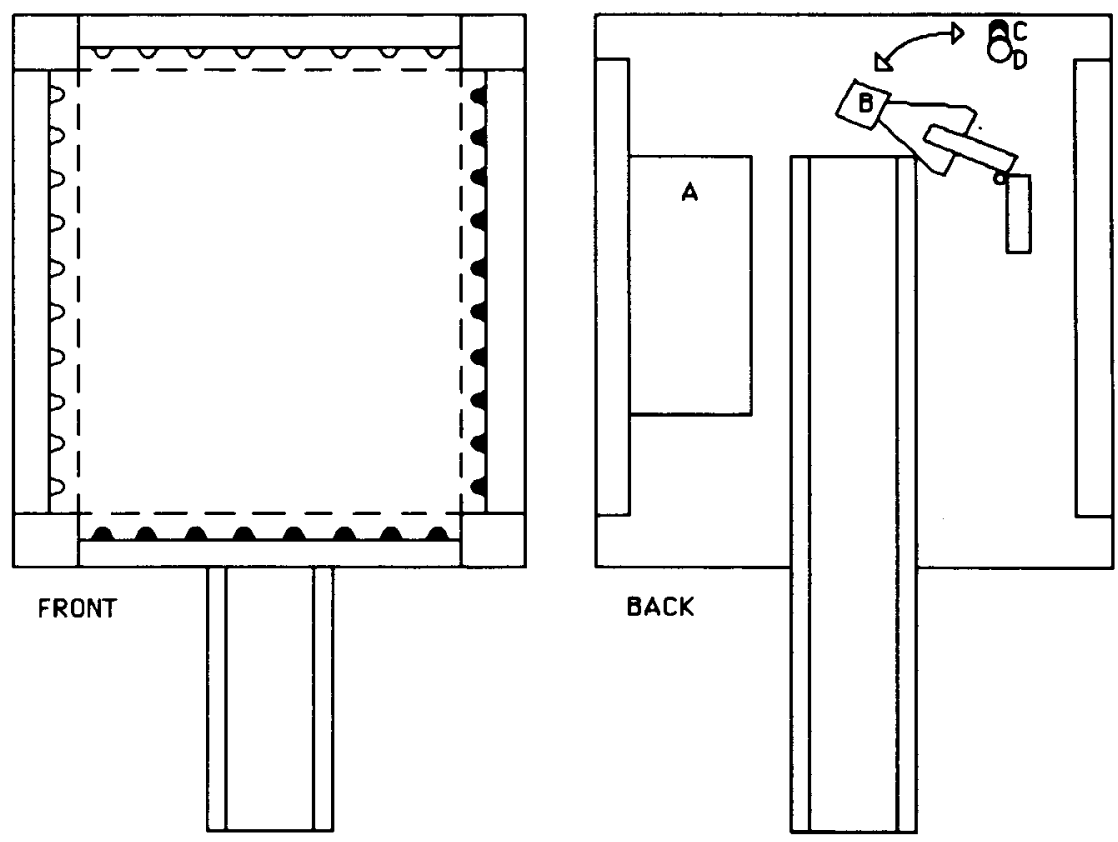

Figure 4. Front and back views of the paddle used to provide location of the ball-paddle contact position, as well as response and movement times. FRONT: Open symbols represent transmitter LEDs and closed symbols represent receiver LEDs. BACK: (A) Circuit board; (B) pendulum end to interrupt infrared beam of tilt sensors; ( $C$ and $D)$ infrared emitter and receiver. LEDs were enclosed to block ambient room light. 
output indicated no interruption, and "high" output indicated beam interruption. A complete scan of all sensor transistors was accomplished approximately 10 times between the initial and final beam interruptions; we used a 12-bit A/D converter with a $30-\mu \mathrm{sec}$ conversion time. The spatial and temporal pattern of beam interruptions during scans of all the comparators was used to compute the time and location of the ball-paddle contact. A flow

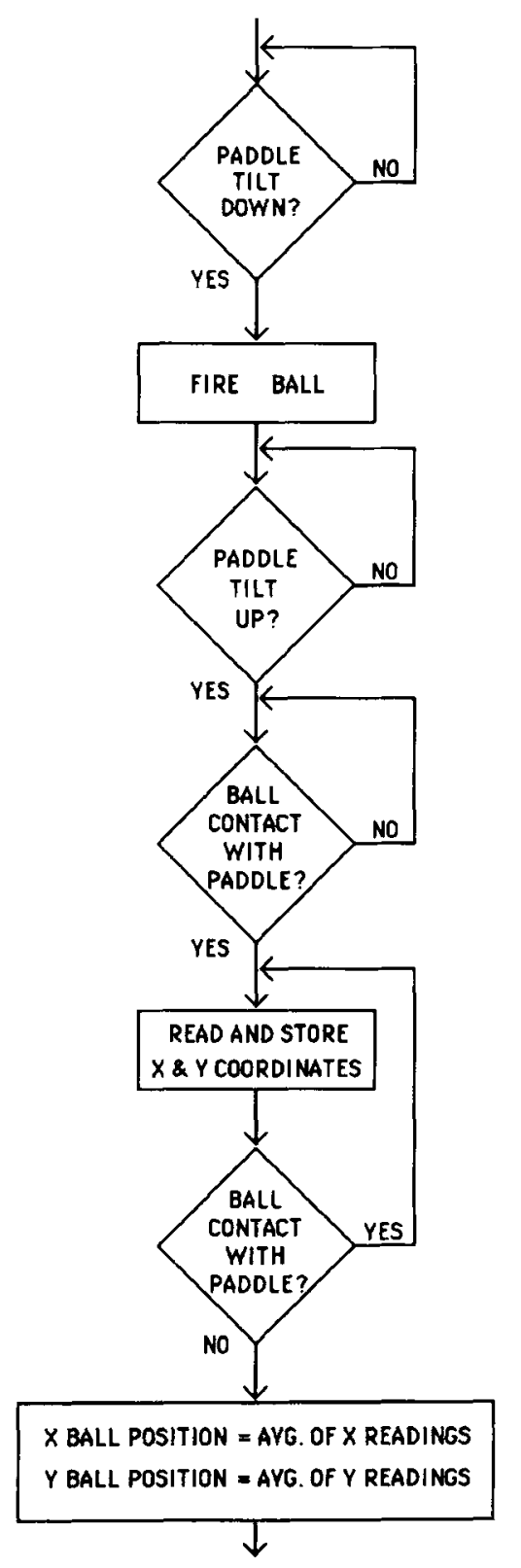

Figure 5. Flow diagram of the program segment controlling ball launch and scan of the analog comparators to determine time and position of ball-paddle contact. diagram of this program segment is shown in Figure 5. The block diagram and schematic of the pendulum, paddle, and scan circuitry are presented in Figures 6 and 7 , respectively.

Applications of the devices can be extended to other experimental situations. The grid of infrared light beams could be used to indicate exocentric target location in pointing or throwing tasks, as well as to indicate egocentric relationship of ball-paddle contact. The target could be used alone or in conjunction with the paddle to monitor the relationships between spatial location of the ballpaddle contact and the target. The intensity of the LEDs used in our paddle limits the size of the detecting surface. Larger targets could be made by focusing the beams with lenses, using higher output LEDs, and/or more sensitive receivers.

\section{Occluders}

Visual input in ball-hitting or ball-catching tasks has been manipulated by extinguishing room lights to preclude view of portions of the ball's flight path (e.g., Whiting $\&$ Sharp, 1974). The time required for light or dark adaptation between trials may pose difficulties for some experimental designs. Other research employed stationary obstacles that interfered with the observers' view of the flight path or of the effector (DeLucia \& Cochran, 1985; Fischman \& Schneider, 1985; McLeod et al., 1985; Smyth \& Marriott, 1982). These methods required a design in which all the trials of one level of an independent variable were presented sequentially. Such a blocked design could encourage different visual-motor strategies for each level. On the other hand, our device allows randomization of the order of visual occlusion, encouraging a general ball-hitting strategy to encompass all stimulus situations provided to the observer.

The computer-controlled shutters allowed left monocular, right monocular, or binocular viewing during task performance. The subjects wore spectacles frames mounted with a small rotary motor $(2.5 \mathrm{~cm}$ in diameter and $2.7 \mathrm{~cm}$ long) on each earpiece. A stiff hemi-elliptical cardboard shutter $(11.4 \mathrm{~cm}$ wide at eye level, and $8.9 \mathrm{~cm}$ long) was attached to each motor. When both shutters were rotated over the eyes, $120^{\circ}$ of the central visual field was occluded. The apparatus weighed $132 \mathrm{~g}$.

Computer control of the motors allowed trial-to-trial changes. A fixed pulse ( $9 \mathrm{~V}, 1,000 \mathrm{msec}$ in duration) to each motor rotated the shutter over an eye. Movement ended when a shutter encountered a small stop mounted on the frame. Speed of shutter rotation was not a relevant factor in our experiment; the maximum rate was twice per second. If desired, quicker shutter rotation could be accomplished by decreasing the weight of the occluding material, by increasing the voltage to the motors, and/or by employing more powerful motors. ${ }^{3}$

Figures 8 and 9 present the block diagram and schematic, respectively, of the control circuitry for the visual occluders. 


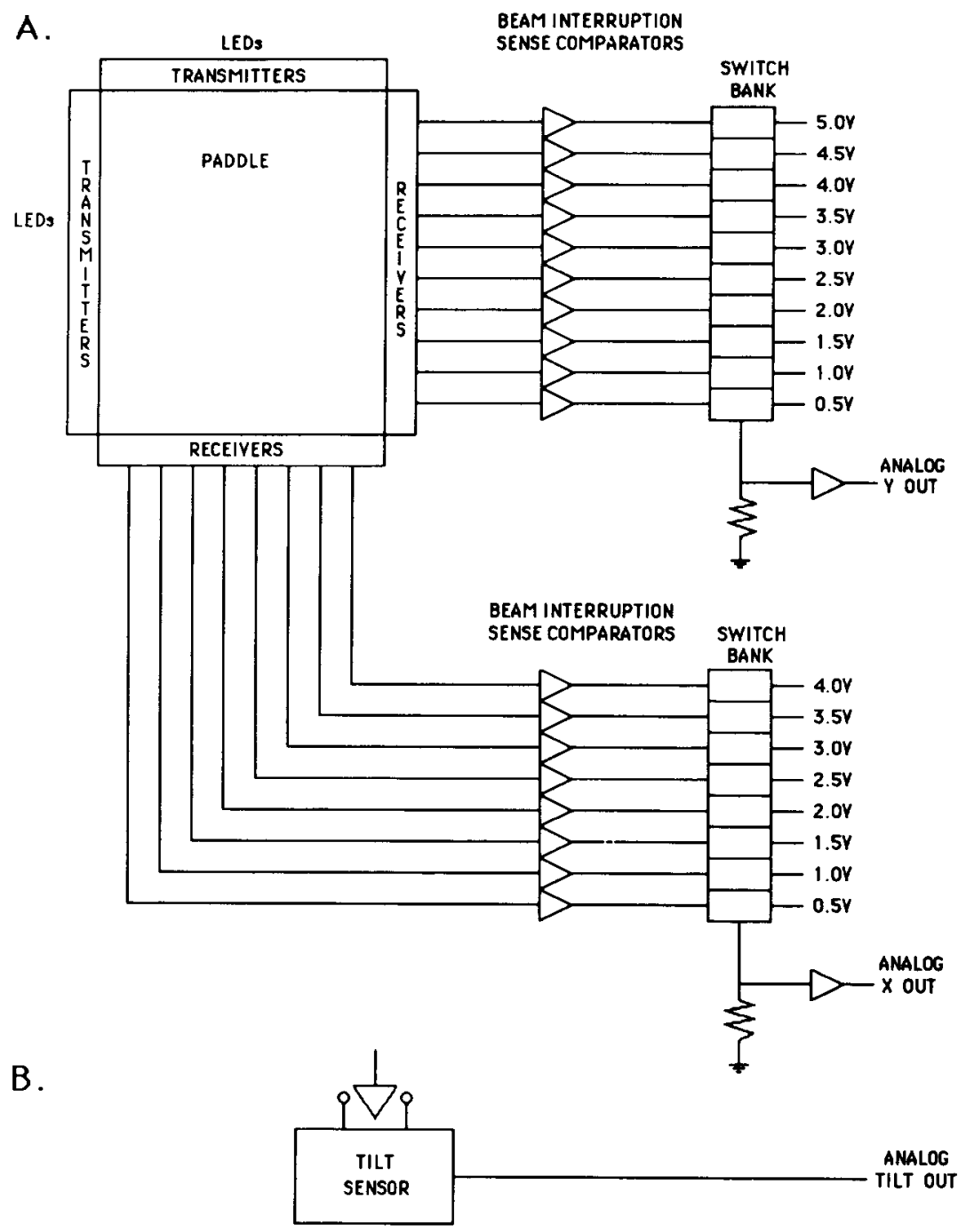

Figure 6. Block diagrams of the paddle. (A) Paddle scan circuit. (B) Pendulum circuit.

To select other aspects of the optical input, one could, for example, replace the cardboard shutters with optic filters or change the size and shape of the shutters to occlude different portions of the visual field (Davids, 1988; Paillard, 1982). In addition, circuitry can be added to allow remote control of the shutters.

Other uses of the shutters include visual occlusion during pointing (e.g., Elliot \& Allard, 1985; Paillard, 1982), hitting (e.g., DeLucia \& Cochran, 1985; McLeod et al., 1985), catching (e.g., Fischman \& Schneider, 1985; Whiting \& Sharp, 1974), throwing (e.g., Eby \& Loomis, 1987), or locomotion (Assaiante, Marchand, \& Amblard, 1989; Elliot, 1986; Thomson, 1983) tasks to determine temporal and spatial aspects of the optic array important for perception and action.

\section{CONCLUSION}

An inexpensive and flexible means of launching tabletennis balls, occluding input to the eyes, and collecting ball-hitting responses was presented. Modification of the circuits to accept digital or analog control or to operate under different parameters could be accomplished by anyone with rudimentary electronic skills.

The electromechanical devices can be used to investigate mechanisms underlying perceptual-motor coordination, skill acquisition, and motor behavior in a variety of action categories. Inexpensive components and automatic stimulus presentation and data acquisition allow analyses of perceptual-motor components previously hindered by extensive and expensive off-line description and analysis. 
A.

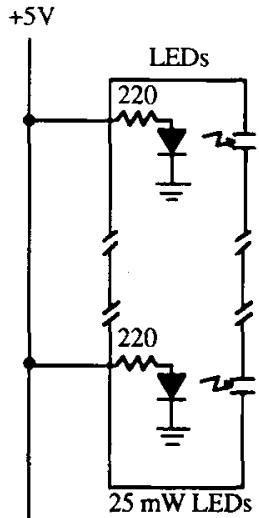

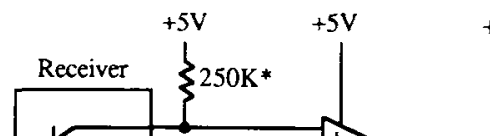

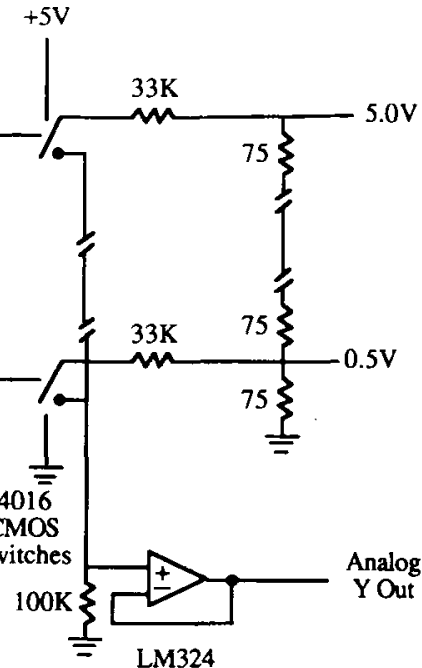

Infrared Phototransistor

Op Amps

4016
CMOS
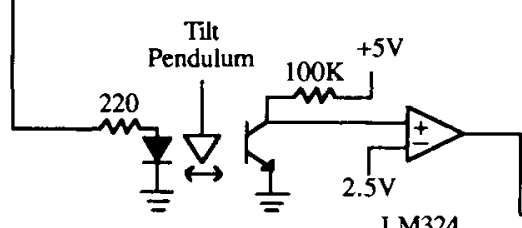

LM324

B.

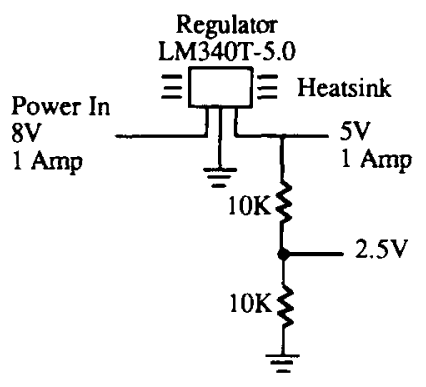

Figure 7. (A) Schematic for the circuit used to scan the infrared light grid for beam interruption. Note that only a portion of the $Y$ circuit is shown. The $X$ circuit, not shown, is similar to the $Y$ circuit. Please see Figure 6 for the block diagram of the complete circuit. $* 250 \mathrm{K \Omega}$ is a typical value. Each sensor resistor, between $75 \mathrm{~K} \Omega$ and 1.5 MO, must be individually determined dependent on the LED-receiver combination. The tilt sensor transmitted the state of the pendulum to the computer. (B) The LM340T supplied regulated $5 \mathrm{~V}$ to the paddle scan circuit.

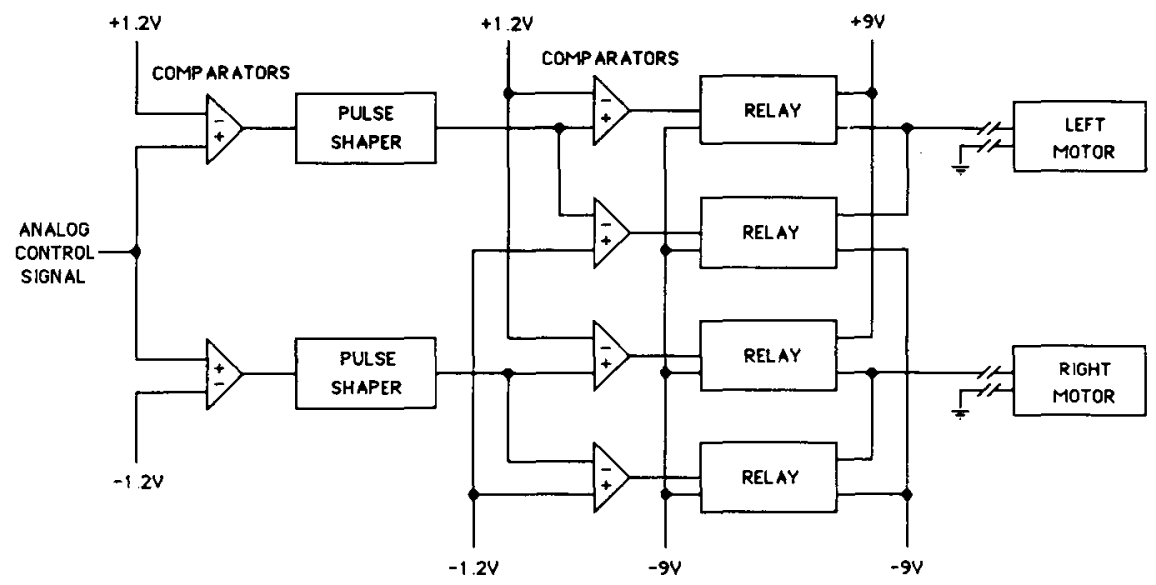

Figure 8. Block diagram of the circuit controlling the visual occluders to allow left monocular, right monocular, or binocular input. 


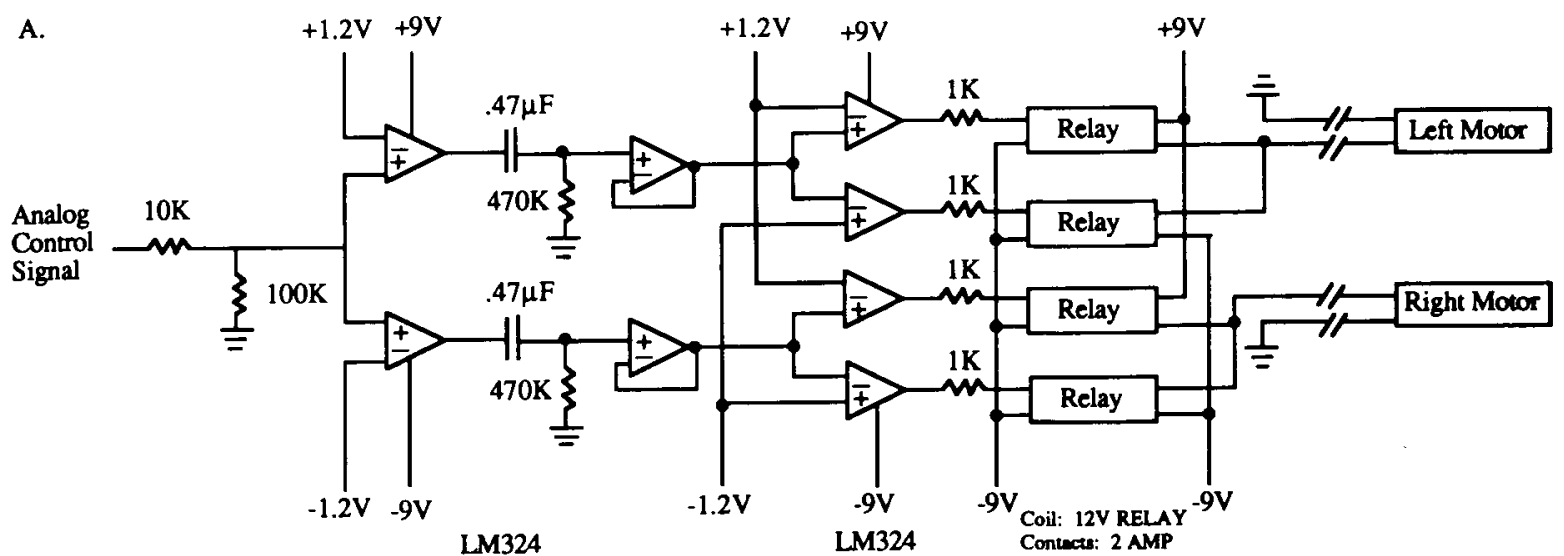

B.

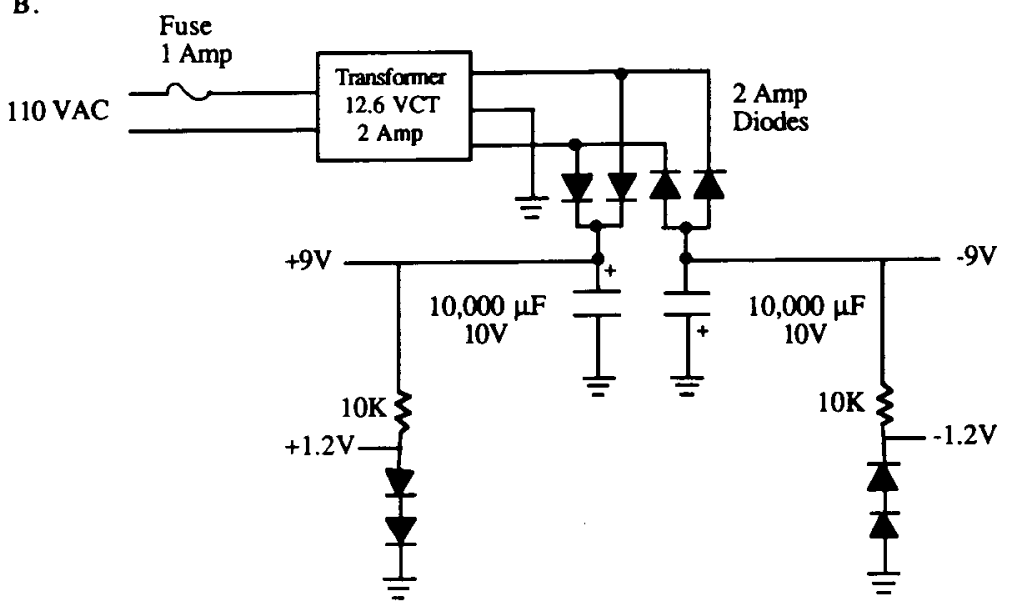

Figure 9. Schematic for the circuit used to control the occluders. Input voltage of $-5.0 \mathrm{~V}, 0.0 \mathrm{~V}$, and $+5.0 \mathrm{~V}$ resulted in, respectively, left shutter open, left and right shutters open, and right shutter open. Note-We could have controlled the relays directly with digital outputs to provide the motors with the power pulse. We chose this method due to constraints of our computer interface.

\section{REFERENCES}

Assainnte, C., Marchand, A. R., \& Amblard, B. (1989). Discrete visual samples may control locomotor equilibrium and foot positioning in man. Journal of Motor Behavior, 21, 72-91.

Bootsma, R. J., \& VAN WIERINGEN, P. C. W. (1988). Visual control of an attacking forehand drive in table tennis. In $O$. J. Meijer \& K. Roth (Eds.), Complex movement behaviour: 'The' motor-action controversy (pp. 189-199). Amsterdam: North-Holland.

Bootsma, R. J., van Wirringen, P. C. W. (1990). Timing an attacking forehand drive in table tennis. Journal of Experimental Psychology: Human Perception \& Performance, 16, 21-29.

Bridgeman, B., Kirch, M., \& Sperling, A. (1981). Segregation of cognitive and motor aspects of visual function using induced motion. Perception \& Psychophysics, 29, 336-342.

Bridgeman, B., LewIS, S., Heit, G., \& Nagle, M. (1979). Relation between cognitive and motor-oriented systems of visual position perception. Journal of Experimental Psychology, 5, 692-700.

Carlton, L. G. (1981). Processing visual feedback information for movement control. Journal of Experimental Psychology: Human Perception \& Performance, 7, 1019-1030.

DAvids, K. (1988). Developmental differences in the use of peripheral vision during catching performance. Joumal of Motor Behavior, 20 , 39-51.
Delucia, P. R., \& Cochran, E. L. (1985). Perceptual information for batting can be extracted throughout a ball's trajectory. Perceptual \& Motor Skills, 61, 143-150.

EвY, D. W., LOOMIS, J. M. (1987). A study of visually directed throwing in the presence of multiple distance cues. Perception \& Psychophysics, 41, 308-312.

Elliot, D. (1986). Continuous visual information may be important after all: A failure to replicate Thomson (1983). Journal of Experimental Psychology: Human Perception \& Performance, 12, 388-391.

Elliot, D., \& Allard, F. (1985). The utilization of visual feedback information during rapid pointing movements. Quarterly Journal of Experimental Psychology, 37A, 407-425.

Fischman, M. G., Schneider, T. (1985). Skill level, vision, and proprioception in simple one-hand catching. Journal of Motor Behavior, 17, 219-229.

.Franks, I. M., Weicker, D., \& Robertson, D. G. E. (1985). The kinematics, movement phasing and timing of a skilled action in response to varying conditions of uncertainty. Human Movement Science, 4, 91-105.

Hansen, R. M., \& Skavenski, A. A. (1985). Accuracy of spatial localizations near the time of saccadic eye movements. Vision Research, 25, 1077-1082.

Keele, S. W., \& Posner, M. I. (1968). Processing of visual feedback in rapid movements. Journal of Experimental Psychology, 77, 155-158. 
LEE, D. N., \& YoUNG, D. S. (1984). Visual timing of interceptive action. In D. J. Ingle, M. Jeannerod, \& D. N. Lee (Eds.), Brain mechanisms of spatial vision (pp. 1-30), La Haye: Martinus Nijhof.

Lee, D. N., Young, D. S., Reddish, P. E., Lough, S., \& Clayton, T. M. H. (1983). Visual timing in hitting an accelerating ball. Quarterly Journal of Experimental Psychology, 35A, 333-346.

Mcleod, P., Mclaughlin, C., \& Nimmo-Smith, I. (1985). Information encapsulation and automaticity: Evidence from the visual control of finely timed actions. In M. I. Posner \& O. M. Marin (Eds.), Attention and performance XI (pp. 391-406). Hillsdale, NJ: Erlbaum.

Milgram, P. (1987). A spectacle-mounted liquid-crystal tachistoscope. Behavior Research Methods, Instruments, \& Computers, 19, 449-456.

Mollon, J. D., Polden, P. G., \& Morgan, M. J. (1977). Electrooptic shutters and filters. Quarterly Journal of Experimental Psychology, 29, 147-156.

Nemire, K. E. (1990). Visual and oculomotor contributions in a ballhitting task. Dissertation Abstracts International, 50, 5908-B.

Paillard, J. (1980). The multichanneling of visual cues and the organization of a visually guided response. In G. E. Stelmach \& J. Requin (Eds.), Tutorials in motor behavior (pp. 259-279). Amsterdam: North-Holland.

Paillard, J. (1982). The contribution of peripheral and central vision to visually guided reaching. In D. J. Ingle, M. A. Goodale, \& R. J. W. Mansfield (Eds.), Analysis of visual behavior (pp. 367-385). Cambridge: MIT Press.

Schmidt, R. A. (1987). Motor control and learning (2nd ed.). Champaign, IL: Human Kinetics.

SHEBILSKE, W. L. (1986). Baseball batters support an ecological efference mediation theory of natural event perception. Acta Psychologica, 63, 117-131.

Smyth, M. M., \& Marriott, A. M. (1982). Vision and proprioception in simple catching. Joumal of Motor Behavior, 14, 143-152.

STARK, L., \& BRIDGEMAN, B. (1983), Role of corollary discharge in space constancy. Perception \& Psychophysics, 34, 371-380.

THOMson, J. A. (1983). Is continuous visual monitoring necessary in visually guided locomotion? Joumal of Experimental Psychology: Human Perception \& Performance, 9, 427-443.

Tyldesley, D. A., WhItiNG, H. T. A. (1975). Operational timing. Journal of Human Movement Studies, 1, 172-177.

Whiting, H. T. A., \& SHaRP, R. H. (1974). Visual occlusion factors in a discrete ball-catching task. Journal of Motor Behavior, 6, 11-16.

Zelaznik, H. N., Hawkins, B., \& Kisselburgh, L. (1983). Rapid visual feedback processing in single-aiming movements. Journal of Motor Behavior, 15, 217-236.

\section{NOTES}

1. The approximate costs of components for the ball delivery system, paddle, and occluders were $\$ 200, \$ 75$, and $\$ 40$, respectively.

2. We subsequently learned that Simplicity Tool Co. (Portland, OR) makes a wonderful device that propels table-tennis balls. Their SitkoTT Robot is appropriate for situations requiring complex ball motions and trajectories encountered during actual game situations. For our initial purposes, though, a simpler ball motion, with little spin, made specification of the ball trajectory a simpler task. It also made hitting the ball an easier task.

3. Selective application of an electric field to liquid-crystal cells mounted in goggles also would produce on-off devices, and would allow randomization of monocular and binocular conditions (Milgram, 1987; Mollon, Polden, \& Morgan, 1977). However, the anticipated costs were too high (about $\$ 2,000-\$ 3,000$ ), and the cells did not allow occlusion for the length of time required for each experimental trial (about $10 \mathrm{sec}$ ). We have recently learned, however, that Milgram now has the capability to increase the extinction time of the liquid-crystal display for any length of time required. The goggles are available, for about $\$ 2,000$, through Translucent Technologies, Inc., in Toronto.

(Manuscript received April 20, 1990; revision accepted for publication December 13, 1990.) 